\title{
Article
}

\section{Effect of Surface Polarity on the Structure and Dynamics of Water in Nanoscale Confinement}

Santiago Romero-Vargas Castrillo\#n, Nicola\#s Giovambattista, Ilhan A. Aksay, and Pablo G. Debenedetti J. Phys. Chem. B, 2009, 113 (5), 1438-1446• DOI: 10.1021/jp809032n • Publication Date (Web): 14 January 2009

Downloaded from http://pubs.acs.org on March 20, 2009

\section{More About This Article}

Additional resources and features associated with this article are available within the HTML version:

- Supporting Information

- Access to high resolution figures

- $\quad$ Links to articles and content related to this article

- Copyright permission to reproduce figures and/or text from this article

\section{View the Full Text HTML}




\title{
Effect of Surface Polarity on the Structure and Dynamics of Water in Nanoscale Confinement
}

\author{
Santiago Romero-Vargas Castrillón, Nicolás Giovambattista, ${ }^{\dagger}$ Ilhan A. Aksay, and \\ Pablo G. Debenedetti*
}

Department of Chemical Engineering, Princeton University, Princeton, New Jersey 08544-5263

Received: October 12, 2008; Revised Manuscript Received: December 9, 2008

\begin{abstract}
We present a molecular dynamics simulation study of the structure and dynamics of water confined between silica surfaces using $\beta$-cristobalite as a model template. We scale the surface Coulombic charges by means of a dimensionless number, $k$, ranging from 0 to 1 , and thereby we can model systems ranging from hydrophobic apolar to hydrophilic, respectively. Both rotational and translational dynamics exhibit a nonmonotonic dependence on $k$ characterized by a maximum in the in-plane diffusion coefficient, $D_{\|}$, at values between 0.6 and 0.8 , and a minimum in the rotational relaxation time, $\tau_{\mathrm{R}}$, at $k=0.6$. The slow dynamics observed in the proximity of the hydrophobic apolar surface are a consequence of $\beta$-cristobalite templating an ice-like water layer. The fully hydrophilic surfaces $(k=1.0)$, on the other hand, result in slow interfacial dynamics due to the presence of dense but disordered water that forms strong hydrogen bonds with surface silanol groups. Confinement also induces decoupling between translational and rotational dynamics, as evidenced by the fact that $\tau_{\mathrm{R}}$ attains values similar to that of the bulk, while $D_{\|}$is always lower than in the bulk. The decoupling is characterized by a more drastic reduction in the translational dynamics of water compared to rotational relaxation.
\end{abstract}

\section{Introduction}

Physical systems in which a confining geometry is in contact with water play an important role in many situations of scientific and technological relevance. A prominent example is the living cell, in which water, present in interstices of $\sim 2 \mathrm{~nm}^{1}$ between biomolecules, regulates the rate of hydrophobic collapse leading to protein folding, ${ }^{2-4}$ and the transport of molecules and ions across biological channels, ${ }^{5,6}$ among other functions. The understanding of water in nanoscopic confinement is also critical for the successful implementation of nanofluidic devices, ${ }^{7-9}$ as well as the design of self-assembling molecular structures ${ }^{10}$ used in nanomaterials synthesis. ${ }^{11}$

In discussing water in nanoscale confinement, we refer to two situations: (i) interfacial water, and (ii) confined water. Interfacial water is commonly understood as being found between a fluid phase-usually its bulk vapor or liquid-and a solid substrate or different fluid phase, while confined water exists between two solid surfaces. The host of interesting phenomena associated with confined and interfacial water can be attributed to a combination of several factors that modify water's thermodynamics and dynamics relative to the bulk. These factors are the size and shape of the confining medium-i.e., the confinement length scale-and the interactions of the fluid with the confining boundaries. ${ }^{12}$ The interplay between confinement (geometric) length scale and fluid correlation length scale, as well as fluid-fluid and fluid-solid interactions, result in a wealth of interesting physical phenomena such as the appearance of phase transitions (e.g., evaporation or dewetting ${ }^{13-15}$ and crystallization ${ }^{16-20}$ ) that would not be observable in the bulk under otherwise identical thermodynamic conditions. The preeminent role of fluid-surface interactions was recently

\footnotetext{
* Corresponding author. E-mail: pdebene@ princeton.edu.

Present address: Physics Department, Brooklyn College of the City University of New York, Brooklyn, NY 11210.
}

demonstrated by Goel et al., ${ }^{21}$ who showed that by manipulating the interactions between a soft-sphere fluid and its confining surfaces, it is possible to tune the fluid's density profile and mobility. Interestingly, the authors found that a highly structured density profile (as opposed to a flat profile) results in a higher diffusion coefficient because of the higher excess entropy of the structured fluid.

The abundance and importance of interfacial and confined water have motivated numerous experimental studies pertaining to its structure and dynamics. A review of experimental, theoretical, and computational studies of water dynamics near proteins and micelles can be found in ref 10. Salmerón and collaborators ${ }^{22}$ and Shen and Ostroverkhov ${ }^{23}$ recently reviewed the state of the art in experimental studies on the structure of interfacial water. Experimental techniques such as nuclear magnetic resonance (NMR), dielectric relaxation, and quasielastic neutron scattering (QENS) have shown that water in the proximity of proteins and micelles exhibits an average mobility that is lower than its bulk counterpart. ${ }^{10}$ However, since these techniques average a response over all molecules, ${ }^{24}$ they are unable to reflect the changes undergone by dynamic properties as they transition from the bulk toward the hydration layer. Moreover, the extent to which confinement affects water dynamics is a matter of much contention. An example of the ongoing debate is found in intracellular water, where, depending on the experimental technique-in vivo NMR vis-à-vis QENSdifferent groups have concluded that most water molecules in living cells are bulk-like, ${ }^{25}$ while others argue that $\sim 76 \%$ of cell water exhibits dynamics that are much slower than those of the bulk. ${ }^{26}$

Other techniques such as atomic force microscopy (AFM) and scanning tunneling microscopy (STM), although intrusive, have proven useful for thin water films on solid surfaces, but less successful at liquid interfaces, while X-ray photoelectron spectroscopy (XPS), X-ray absorption, and attenuated total 
reflection (ATR) infrared spectroscopy have large penetration lengths ( $\geq 1 \mathrm{~nm}$ and $\geq 100 \mathrm{~nm}$, respectively) that hinder the study of the buried interface. ${ }^{23}$ Nonetheless, emerging experimental techniques such as sum-frequency vibrational spectroscopy (SFVS), ${ }^{23,27}$ and femtosecond mid-infrared spectroscopy ${ }^{24}$ hold promise as local probes of water in the (first) hydration layer. Recently, Rezus and Bakker ${ }^{24}$ reported what appears to be the first direct proof of the existence of immobilized water molecules in the hydration layer of hydrophobes, thereby confirming certain aspects of the "iceberg" model proposed by Frank and Evans more than 60 years ago. ${ }^{28}$

Despite the considerable progress shown by different experimental techniques, molecular simulations continue to be a powerful tool for studying interfacial systems, often complementing and guiding the interpretation of experimental results. In particular, molecular dynamics (MD) simulations permit the characterization of local structural and dynamic properties which, assuming accurate force fields, result in molecular-level information that is difficult, if not impossible, to access experimentally. MD simulations have enabled the determination of local structure and dynamics of a wide array of confined and interfacial water systems. ${ }^{18,29-32}$ Recent examples of MD studies in thin water films include the work of Liu et al., ${ }^{33}$ who studied the hydrogen bond (HB) and translational dynamics of water as a function of distance from the vapor-liquid interface, and the study of Giovambattista et al. ${ }^{34}$ on the effect of surface polarity on water molecule orientation at the interface with silica ( $\beta$-cristobalite) surfaces. Simulation studies have also shown that HB dynamics substantially slow down near polar or charged head groups of micelles and proteins, ${ }^{10,35}$ as well as in the proximity of water-organic liquid interfaces. ${ }^{36}$

Given that surface structure and polarity largely determine the dynamic properties of water molecules near surfaces, ${ }^{10,18,37}$ there is a definite need for systematic simulation studies focusing on the effect of these structural properties on the translational and rotational dynamics of confined water, covering the complete range from apolar to hydrophilic surfaces. In this paper, we extend the understanding of this structure-property relation using MD simulations. Following the formulation of Giovambattista et al., ${ }^{34}$ we scale the dipole moment of $\beta$-cristobalite surfaces through the dimensionless number $k \in[0,1]$ (hydrophobic apolar corresponding to $k=0.0$, while hydrophilic surfaces result when $k=1.0$ ) and study the effect of surface polarity and template structure on the local structural and dynamic properties of confined water. We find that the dynamics evolve nonmonotonically with $k$, with a maximum in the inplane translational diffusion coefficient, $D_{\|}$, appearing at $k \in$ $[0.6,0.8]$ and a minimum in the rotational relaxation time, $\tau_{\mathrm{R}}$, at $k=0.6$. We explain these observations as the result of several phenomena occurring near the confining surfaces: the formation of ice-like structures on apolar $\beta$-cristobalite, an effect which appears to be specific to the silica structure considered in this work; the disruption of the aforementioned structures upon surface hydrogenation, causing the maxima in rotational and translational dynamics $(k=0.6,0.8)$; and the formation of dense but disordered water on the surfaces when $k=1.0$. Our analysis of local dynamics also presents evidence for the decoupling of rotational and translational dynamics.

This paper is structured as follows. In section 2 we present the simulation details. Section 3 contains our results on translational and rotational dynamics and on structural properties. Finally, in section 4 we present the conclusions drawn from this study, as well as directions for future inquiry.

\section{Simulation and Computational Details}

We study the structure and dynamics of confined water using MD simulations in the canonical (constant $N, V, T$ ) ensemble. The domain temperature is set to $300 \mathrm{~K}$ using a Berendsen thermostat ${ }^{38}$ and water mean density is fixed at $1.0 \mathrm{~g} \mathrm{~cm}^{-3}$. The system geometry consists of a slab of water confined between two $\beta$-cristobalite plates. We apply periodic boundary conditions in the $x$ and $y$ directions, making the system macroscopic in the directions parallel to the surfaces and confined in the $z$ direction. We use the extended simple-point-charge (SPC/E) pair potential, ${ }^{39}$ which has been shown to reproduce the experimental rotational and translational dynamic properties of water better than several classical force fields, ${ }^{40}$ including the SPC, ${ }^{41}$ TIP3P, ${ }^{42}$ TIP4P ${ }^{42} \mathrm{SPC} / \mathrm{RF},{ }^{40}$ and TIP4P/RF ${ }^{40}$ models. SPC/E is a rigid three-site model in which the $\mathrm{H}-\mathrm{O}-\mathrm{H}$ angle is $109.47^{\circ}$ and the $\mathrm{O}-\mathrm{H}$ distance is $0.1 \mathrm{~nm}$. Each atom in a SPC/E water molecule is assigned a point charge $\left(q_{\mathrm{H}}=0.4238 e, q_{\mathrm{O}}=-2 q_{\mathrm{H}}\right)$. In addition, the oxygen atom bears a Lennard-Jones interaction site with $\sigma=0.31656 \mathrm{~nm}$ and $\epsilon=0.65017 \mathrm{~kJ} \mathrm{~mol}^{-1}$. The initial configuration of water molecules is obtained from MD simulations of bulk SPC/E water. Further details on the system have been published elsewhere. ${ }^{18}$

Each confining silica wall $\left(6.93 \times 6.93 \mathrm{~nm}^{2}, 0.866 \mathrm{~nm}\right.$ thick $)$ reproduces the (111) plane of $\beta$-cristobalite. ${ }^{18} \alpha$-Cristobalite is the most stable phase at the temperature at which we conduct our simulations. ${ }^{43}$ Nevertheless, we choose to model the metastable $\beta$-cristobalite phase because an accurate force field for this type of surface has been developed previously. ${ }^{29}$ As part of an ongoing research effort we plan to investigate the effects of changing the surface structure to that of the stable phase, $\alpha$-cristobalite, in addition to amorphous silica and silica gels; as well as the inclusion of surface defects, which have been shown to affect water adsorption. ${ }^{44}$ We will also study the effect of substrate flexibility (vide infra).

The silica wall unit cells consist of $\mathrm{SiO}_{4}$ tetrahedra, in which the $\mathrm{Si}-\mathrm{O}$ and $\mathrm{O}-\mathrm{O}$ distances are 0.151 and $0.247 \mathrm{~nm}$, respectively. $\mathrm{Si}$ and $\mathrm{O}$ atomic vibrations have not been taken into account in our simulations. This implementation is strongly preferred since our objectives included the characterization of confined water compatible with a given substrate geometry, a clear answer to which is confounded by substrate relaxation. Moreover, a Lindemann-type estimate based on the lattice constant of our $\beta$-cristobalite surfaces $(0.494 \mathrm{~nm})$ shows that the magnitude of $\mathrm{Si}$ and $\mathrm{O}$ atomic vibrations should be $\ll \sim 0.05$ $\mathrm{nm}$ (i.e., the melting threshold, approximately equal to $10 \%$ of the lattice constant). ${ }^{18}$ This is more than an order of magnitude smaller than the smallest wall-wall separation investigated $(d$ $=1.0 \mathrm{~nm}$, vide infra), suggesting that substrate relaxation has a small effect and does not significantly affect our results. Problems in which substrate conformational changes should be fully accounted for, and dissociable or ab initio potentials used, such as the chemistry of water at silica interfaces, are beyond the scope of our study.

We consider both hydroxylated and non-hydroxylated silica surfaces. In the latter, the $\mathrm{Si}$ and $\mathrm{O}$ atoms possess interaction sites that are exclusively of the Lennard-Jones type. The hydroxylated surfaces are constructed by attaching a hydrogen atom to the surface oxygen, thereby forming a surface silanol group on the side of the wall in contact with water. The $\mathrm{O}-\mathrm{H}$ distance is set to $0.1 \mathrm{~nm}$ and the $\mathrm{Si}-\mathrm{O}-\mathrm{H}$ angle to $109.47^{\circ} .{ }^{18}$ Surface hydrogens can reorient in a circle on a plane parallel to the surface, located $0.033 \mathrm{~nm}$ above the surface oxygens. In addition to the $\mathrm{Si}$ and $\mathrm{O}$ Lennard-Jones interactions, the surface $\mathrm{Si}, \mathrm{O}$ and $\mathrm{H}$ possess a partial charge modeling the dipole 
TABLE 1: Potential Parameters for Surface-Water Interactions $^{a}$

\begin{tabular}{cccc}
\hline atom & $\epsilon\left(\mathrm{kJ} \mathrm{mol}^{-1}\right)$ & $\sigma(\mathrm{nm})$ & charge (e) \\
\hline $\mathrm{O}$ & 0.6487 & 0.3154 & -0.71 \\
$\mathrm{Si}$ & 0.5336 & 0.3795 & 0.31 \\
$\mathrm{H}$ & & & 0.40 \\
${ }^{a}$ Reference 29. & & &
\end{tabular}

moment of the silanol group. Long-range electrostatic interactions are handled with the Ewald sum method, using a cutoff distance of $0.79 \mathrm{~nm}$ and parameters $m_{\max }=5$ (for the number of vectors in the reciprocal-space sum) and $\alpha=4.0 \mathrm{~nm}^{-1}$ (for the width of the screening-charge Gaussian distribution). ${ }^{18}$ Table 1 presents the potential parameters for surface water interactions. $^{18,29}$

We explore two wall-wall separations, $d=1.0$ and $1.6 \mathrm{~nm}$ (where the wall-wall distance is defined as that between the planes containing the surface hydrogen atoms). We place 1605 or $2568 \mathrm{SPC} / \mathrm{E}$ water molecules between the plates, depending on the separation, obtaining the target mean density of $1.0 \mathrm{~g}$ $\mathrm{cm}^{-3}$.

Surface polarity is tuned following ref 34 . In real systems, surface electrostatics cannot be varied without also modifying other surface structural variables (e.g., atomic size). This precludes a truly systematic investigation of the effect of surface polarity since two (or more) variables are changed at the same time. The scheme devised in ref 34 and explained below enables us to uncover the effect of surface polarity while keeping constant all other variables affecting the properties of confined water.

The surface dipole moment is a result of the contribution of the polar $\mathrm{Si}-\mathrm{O}$ and $\mathrm{O}-\mathrm{H}$ bonds, each of which is characterized by dipole moments $\vec{p}_{\mathrm{SiO}}$ and $\vec{p}_{\mathrm{OH}}$. Hence the total dipole moment of a silanol group, $\vec{p}$, is given by

$$
\vec{p}=\vec{p}_{\mathrm{SiO}}+\vec{p}_{\mathrm{OH}}
$$

where

$$
\begin{aligned}
& \vec{p}_{\mathrm{SiO}}=k\left(q_{\mathrm{Si}} \vec{r}_{\mathrm{SiO}}\right) \\
& \vec{p}_{\mathrm{OH}}=k\left(q_{\mathrm{H}} \vec{r}_{\mathrm{HO}}\right)
\end{aligned}
$$

where $q_{i}$ represents the point charge of atom $i$ (see Table 1) and the vectors $\vec{r}_{i j}=\vec{r}_{i}-\vec{r}_{j}$. The parameter $k$ multiplies the charges of surface $\mathrm{Si}, \mathrm{O}$ and $\mathrm{H}$ atoms, providing a means to tune the surface dipole and change the nature of the surface from apolar hydrophobic to hydrophilic. When $k=0.0$, all surface charges are multiplied by 0 and surface water interactions are exclusively of the Lennard-Jones type. Conversely, when $k=1.0$, the water-surface interactions are modeled with Lennard-Jones and electrostatic interactions, using the parameters in Table 1 . In this study we vary $k$ in intervals of 0.2 . The simulations are run for $1.0 \mathrm{~ns}$, saving configurations every 1.0 ps. In all cases we discard the first 100 ps in order to ensure that the system is at equilibrium.

\section{Results and Discussion}

\subsection{Dynamics of Confined Water.}

3.1.1. Translational Dynamics. Translational dynamics are usually quantified in terms of a diffusion coefficient. In MD

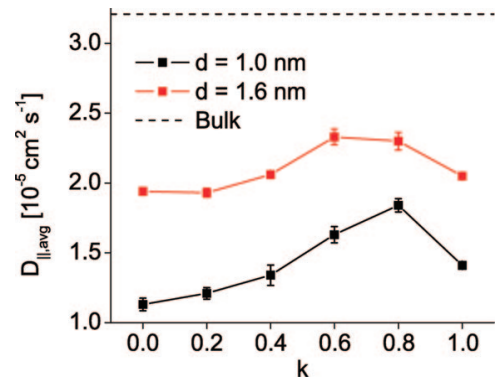

Figure 1. Average in-plane diffusion coefficient as a function of surface polarity $(k)$ and wall-wall separation $(d) . T=300 \mathrm{~K}, \rho=$ $1.0 \mathrm{~g} \mathrm{~cm}^{-3}$.

simulations this property is calculated from a numerical implementation of the Einstein relation, ${ }^{45}$ in which the molecule or atom mean-squared displacement (MSD) is computed as a function of time. The diffusion coefficient is then obtained from the long-time limit of the slope of the resulting straight line. ${ }^{45}$ When the Einstein relation is used to compute the diffusion coefficient of a confined fluid, the underlying assumption of fluid homogeneity is not satisfied given the extensive layering caused by the confining surfaces. ${ }^{46}$ Nonetheless, one can make use of the Einstein relation to compute an in-plane (parallel to the confining surfaces) diffusion coefficient, bearing in mind that this quantity reflects particle mobility averaged over regions characterized by different dynamics.

We compute the MSD of the water oxygen atoms parallel to the confining surfaces, $\left\langle\left(x-x_{0}\right)^{2}+\left(y-y_{0}\right)^{2}\right\rangle$, where the angled brackets denote averaging over all molecules and starting times, and subsequently the average in-plane diffusion coefficient $D_{\|, \text {avg }}$. The results are given in Figure 1 , which presents $D_{\|, \text {avg }}$ as a function of surface polarity, $k$, and $d$.

Figure 1 shows that the evolution of $D_{\|, \text {avg }}$ with surface polarity is nonmonotonic, with a maximum around $k=0.8$ for $d=1.0 \mathrm{~nm}$ and $k \in[0.6,0.8]$ for $d=1.6 \mathrm{~nm}$. The hindered mobility of water molecules confined by hydrophilic surfaces ( $k=1.0$ ) has been observed with similar confining surfaces and was attributed to the strong HB interactions between water and surface silanol groups. ${ }^{29}$ The slow translational dynamics observed at $k=0.0$, however, seems counterintuitive at first sight given the comparatively weaker water-substrate interactions. Fast interfacial dynamics have been observed for TIP4P and TIP5P water ${ }^{29,47}$ and for a Lennard-Jones fluid ${ }^{48}$ confined by perfectly flat hydrophobic surfaces, while the dynamics of a Lennard-Jones fluid confined by a rough hydrophobic surface appear to slow down. ${ }^{48}$ This suggests that not only surface-fluid interactions but also the surface structure define the interfacial dynamics. In addition to the nonmonotonic trend, we note in Figure 1 that $D_{\|, \text {avg }}<D_{\text {Bulk }}=3.2 \times 10^{-5} \mathrm{~cm}^{2} \mathrm{~s}^{-1}$ for all polarities and separations. Our $D_{\text {Bulk }}$, obtained from a MD simulation of bulk water at the same thermodynamic conditions, is $28 \%$ higher than that reported in the literature for SPC/E, $2.5 \times 10^{-5} \mathrm{~cm}^{2}$ $\mathrm{s}^{-1}$ at $306 \mathrm{~K}$ and $0.998 \mathrm{~g} \mathrm{~cm}^{-3}$, and $33 \%$ higher than the experimental diffusion coefficient of water, $2.4 \times 10^{-5} \mathrm{~cm}^{2} \mathrm{~s}^{-1}$ at $300 \mathrm{~K}^{39}$ It is known that the system size, interaction truncations, and long-range interaction schemes (e.g., reaction field and Ewald sums) affect the structure and dynamics of simulated water. ${ }^{40,49}$ For example, values as high as $4.4 \times 10^{-5}$ $\mathrm{cm}^{2} \mathrm{~s}^{-1}$ have been reported for SPC/E water using the Ewald sum method. ${ }^{50}$ Since the value reported by Berendsen et al. ${ }^{39}$ was obtained applying a spherical cutoff for long-range interactions in a smaller system than ours (216 SPC/E water molecules in Berendsen's work compared to 729 in our bulk simulations), where we use the Ewald summation, we deem it appropriate to 
attribute the discrepancy in $D$ to differences in system size and method of truncation.

We investigate the origin of the nonmonotonic behavior observed in Figure 1 by computing the local in-plane diffusion coefficient $D_{\|}(z)$, i.e., the diffusion coefficient in the $x-y$ plane as a function $z$, the distance from the confining surfaces. To this end, we apply the formalism of Berne and collaborators, ${ }^{46}$ according to which the MSD is computed in thin slabs $0.3 \mathrm{~nm}$ in thickness $(0.35 \mathrm{~nm}$ for $d=1.0 \mathrm{~nm})$ parallel to the surfaces. We divide the confined volume with $d=1.0 \mathrm{~nm}$ into 9 slabs, the centers of which are found at $z= \pm 0.175, \pm 0.225, \pm 0.275$, \pm 0.325 , and $0.5 \mathrm{~nm}$. At a separation of $d=1.6 \mathrm{~nm}$ we use 13 slabs centered at $z= \pm 0.15, \pm 0.25, \pm 0.35, \pm 0.45, \pm 0.55$, \pm 0.65 , and $0.8 \mathrm{~nm}$. Since the system is symmetric, we average the results obtained from slabs placed at the same distance from the surfaces but on opposite sides of the symmetry plane (e.g., at $z=-0.15$ and $z=0.15 \mathrm{~nm}$ ) and heretofore report the average.

The local MSD is computed using the following equation:

$$
\begin{array}{r}
\left\langle\Delta \vec{r}(\tau)^{2}\right\rangle_{\{z\}}=\frac{1}{N(0)} \sum_{i \in \sigma\{z\}}\left[\left(x_{i}(\tau)-x_{i}(0)\right)^{2}+\right. \\
\left.\quad\left(y_{i}(\tau)-y_{i}(0)\right)^{2}\right]
\end{array}
$$

where $\sigma\{z\}$ represents the set of molecules that remain in the slab centered at a distance $z$ from the surfaces during the interval $[0, \tau]$. Note that, with this definition, molecules that leave the slab and subsequently re-enter are not counted in the summation. We then compute the survival probability of molecules in the slab, $P(\tau)$, given by

$$
P(\tau)=\frac{N(0, \tau)}{N(0)}
$$

where $N(0)$ denotes the number of molecules present in the slab at time zero and $N(0, \tau)$ represents those which remain in the slab for the entire time interval $[0, \tau]$. Finally, a modified Einstein relation provides the local in-plane diffusion coefficient. ${ }^{46}$ Plotting $\left\langle\Delta \vec{r}(\tau)^{2}\right\rangle_{\{z\}} / 4 P(\tau)$ vs $\tau$ results in a straight line with a slope equal to $D_{\|}(z)$ :

$$
D_{\|}(z)=\lim _{\tau \rightarrow \infty} \frac{\left\langle\Delta \vec{r}(\tau)^{2}\right\rangle_{\{z\}}}{4 P(\tau) \tau}
$$

Typically, the diffusive regime is attained after $\sim 15 \mathrm{ps}$ in the systems with $d=1.6 \mathrm{~nm}$ in the slabs near the center of the confined volume, and at $t \geq 50 \mathrm{ps}$ for slabs within $0.3 \mathrm{~nm}$ from the surfaces. For the systems with $d=1.0 \mathrm{~nm}$, diffusive dynamics are seen after $\sim 50 \mathrm{ps}$ near the center, and $t \sim 100 \mathrm{ps}$ near the surfaces. Having located the diffusive regime, the slope is computed from the following $10 \mathrm{ps}$ of simulation data. To improve sampling statistics, we average over all initial times.

Figure 2 presents the obtained self-diffusion coefficient as a function of distance from the surfaces, calculated using eq 6 . The error bars were computed using a block-averaging method. ${ }^{51}$ We note that $D_{\|}(z)$ decreases monotonically near the confining surfaces for both separations, an observation that is consistent with previous studies ${ }^{29,52}$ of water confined by atomically detailed surfaces. We further note that for both wall-wall separations $D_{\|}(z)$ in the slab adjacent to the silica surfaces (found at $z=0.175 \mathrm{~nm}$ for the system with $d=1.0 \mathrm{~nm}$ and at $z=$

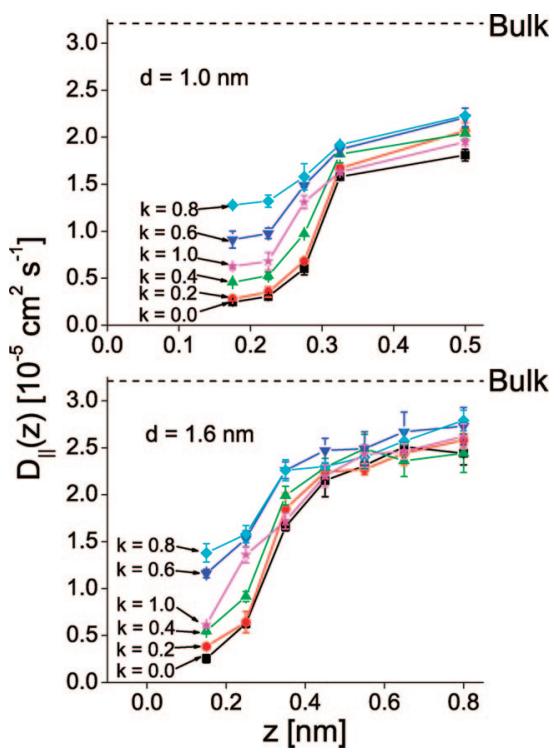

Figure 2. Local in-plane diffusion coefficient of water confined between silica walls as a function of surface polarity $(k)$ and distance from the surfaces, $z \cdot T=300 \mathrm{~K}, \rho=1.0 \mathrm{~g} \mathrm{~cm}^{-3}$. Upper: $d=1.0 \mathrm{~nm}$, lower: $d=1.6 \mathrm{~nm}$.

$0.15 \mathrm{~nm}$ when $d=1.6 \mathrm{~nm}$ ) is roughly an order of magnitude lower than the bulk for $k=0.0,0.2,0.4$, and 1.0. The effect of the surface on water translational dynamics becomes less pronounced away from the plates. Accordingly, all $D_{\|}(z)$ profiles approximately collapse onto the same curve as $z$ increases.

Both panels in Figure 2 show that $D_{\|}(z)<D_{\text {Bulk }}$ throughout the confined volume. Previous studies in which the local dynamics were investigated in confined geometries have found that the diffusion coefficient is bulk-like at the center of the confined volume, albeit at larger confinement length scales. Lee and Rossky ${ }^{29}$ found that TIP4P water translational dynamics are bulk-like at $\sim 2.4 \mathrm{~nm}$ from a fully hydroxylated silica surface. Similarly, Sega et al. ${ }^{52}$ determined that the in-plane diffusion coefficient of SPC water is bulk-like at $\sim 2.0 \mathrm{~nm}$ from a amphiphilic bilayer surface. Liu et al. ${ }^{33}$ found bulk-like translational dynamics of TIP4P/FQ water at a distance of $\sim 1.0$ $\mathrm{nm}$ from the water-vapor interface.

Figure 3 shows the $k$ dependence of $D_{\|}(z)$ in the individual slabs. Local dynamics in the slabs nearest to the surfaces $(z=$ $0.175 \mathrm{~nm}$ for $d=1.0 \mathrm{~nm}$ and $z=0.15 \mathrm{~nm}$ when $d=1.6 \mathrm{~nm}$ ) reflect the nonmonotonic behavior of $D_{\|}(z)$ characterized by a well-defined maximum at $k=0.8$. This nonmonotonic behavior persists in layers not immediately adjacent to the wall: $z \leq 0.275$ $\mathrm{nm}$ when $d=1.0 \mathrm{~nm}$ and $z \leq 0.35 \mathrm{~nm}$ at $d=1.6 \mathrm{~nm}$.

3.1.2. Rotational Dynamics. We analyze rotational dynamics through a local implementation of the dipole moment time correlation function, $C_{1}(\tau)_{\{z\}}$,

$$
C_{1}(\tau)_{\{z\}}=\frac{1}{P(\tau) N(0)} \sum_{i \in \sigma\{z\}} \cos \theta_{i}(\tau)
$$

where the subindex $\{z\}$ indicates that the calculation only considers molecules remaining in the slab centered at $z$ during the interval $[0, \tau]$, and $\theta_{i}$ denotes the angle formed by the dipole moment unit vectors at time 0 and $\tau(\hat{\mu}(0)$ and $\hat{\mu}(\tau)$, respectively) of the $i$ th molecule. The meaning of $P(\tau)$ and $N(0)$ is the same as in equation (5). We compute the local rotational relaxation time, $\tau_{\mathrm{R}}(z)$, by fitting a stretched exponential $\left(A \mathrm{e}^{-\left(\tau / \tau_{\mathrm{R}}(z)\right)^{\beta}}\right)^{53}$ to $C_{1}(\tau)_{\{z\}}$, finding that the decay of $C_{1}(\tau)_{\{z\}}$ is well described by 


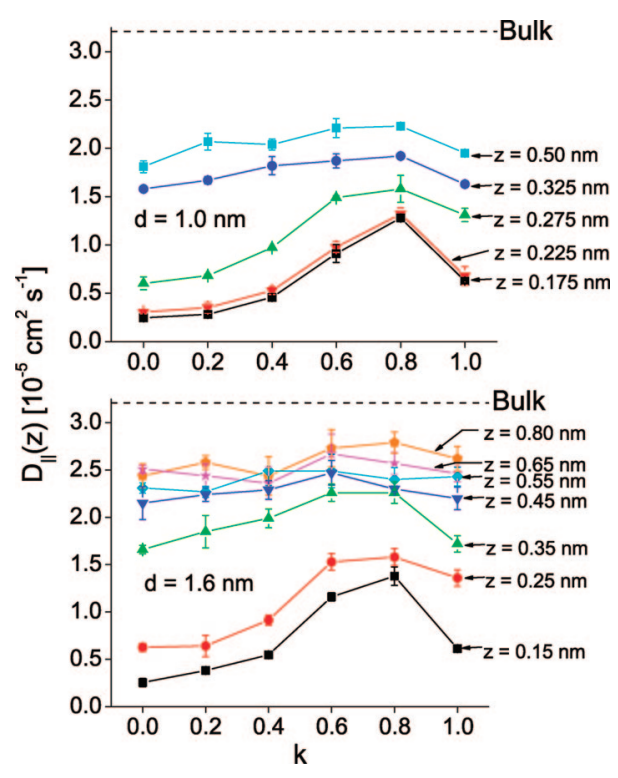

Figure 3. $k$ dependence of the local in-plane diffusion coefficient in various slabs, denoted by the distance from the surfaces, $z . T=300$ $\mathrm{K}, \rho=1.0 \mathrm{~g} \mathrm{~cm}^{-3}$. Upper: $d=1.0 \mathrm{~nm}$, lower: $d=1.6 \mathrm{~nm}$.

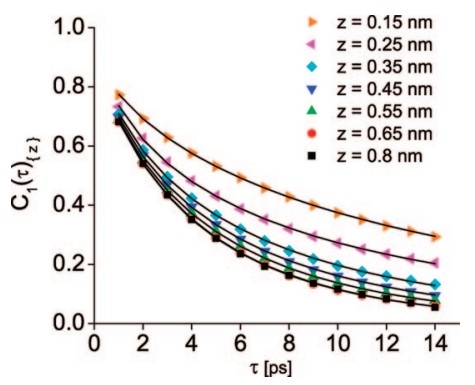

Figure 4. Local time correlation function, $C_{1}(\tau)_{\{z\}}$, as a function of time and distance from the confining surfaces, $z . T=300 \mathrm{~K}, \rho=1.0 \mathrm{~g}$ $\mathrm{cm}^{-3}, d=1.6 \mathrm{~nm}, k=1.0$. Solid black lines indicate the stretched exponential fits.

the above function for times between 1 and 10-40 ps. Figure 4 presents the data corresponding to the system with $k=1.0$ and $d=1.6 \mathrm{~nm}$. Nonlinear regressions resulted in $R^{2} \simeq 0.9998$ or higher. We also compute the rotational relaxation time for bulk water from our MD simulation at $300 \mathrm{~K}$ and $\rho=1.0 \mathrm{~g}$ $\mathrm{cm}^{-3}$, obtaining a value of $4.02 \mathrm{ps}$, in agreement with published data for SPC/E water. ${ }^{40}$

Figure 5 presents the $k$ dependence of $\tau_{\mathrm{R}}(z)$ for different slabs. The error bars denote the $95 \%$ confidence interval of $\tau_{\mathrm{R}}(z)$. Similar to our analysis of translational dynamics, we observe shorter relaxation times away from the surface as well as a nonmonotonic evolution of $\tau_{\mathrm{R}}(z)$ with $k$, manifested by a minimum at $k=0.6$. Furthermore, for $d=1.6 \mathrm{~nm}$, the minimum disappears at $z \geq 0.45 \mathrm{~nm}$, with the two slabs nearest to the center $(z=0.65$ and $0.8 \mathrm{~nm})$ exhibiting bulk-like rotational relaxation times for all $k$ (for clarity, only the slab at $z=0.65$ $\mathrm{nm}$ is shown in Figure 5). A nonmonotonic dependence of HB dynamics on polarity was previously reported by Benjamin ${ }^{36}$ for flexible SPC water at a liquid-liquid interface. The author reports longer water $\mathrm{HB}$ lifetimes at the interface with 1,2dichloroethane, a weakly polar liquid, compared to carbon tetrachloride (an apolar liquid) and nitrobenzene, a polar, HBforming liquid. The occurrence of this maximum was explained by density fluctuations at the water-dichloroethane interface, resulting in water molecules which lack neighboring, nonhydrogen-bonded water molecules. ${ }^{36}$

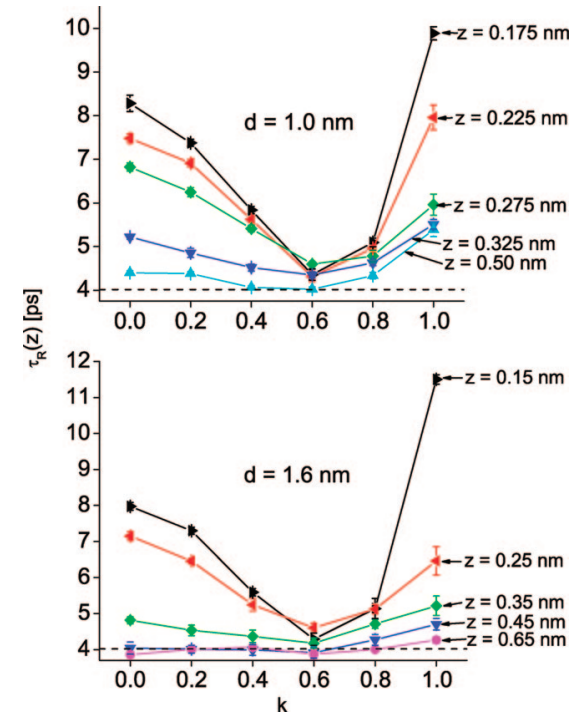

Figure 5. $k$ dependence of the local rotational relaxation time in various slabs, denoted by the distance from the surfaces, $z . T=300 \mathrm{~K}, \rho=$ $1.0 \mathrm{~g} \mathrm{~cm}^{-3}$. Upper: $d=1.0 \mathrm{~nm}$, lower: $d=1.6 \mathrm{~nm}$. The dotted line denotes the bulk value.

The emergence of bulk-like rotational relaxation times is the most important observation in Figure 5: while translational dynamics are slower than those of the corresponding bulk liquid under the same thermodynamic conditions, rotational dynamics at $z \geq 0.65 \mathrm{~nm}$ when $d=1.6 \mathrm{~nm}$ (and also in the $z=0.5 \mathrm{~nm}$ slab when $d=1.0 \mathrm{~nm}$ and $k=0.4,0.6$, cf. upper panel in Figure 5) are approximately bulk-like. This observation points toward a decoupling between rotational and translational dynamics of water in confinement, i.e., that $D_{\|}(z)$ increases more slowly compared to $\tau_{\mathrm{R}}(z)$ away from the surfaces. Computational and experimental studies of supercooled and confined liquids ${ }^{54-58}$ have reported decoupling between rotational and translational dynamics. The translational-rotational decoupling will be further discussed in section 3.4.

Thus far it has been shown that the dynamic properties of water in confinement exhibit a nonmonotonic dependence on surface polarity in both translational and rotational motion, as well as translational-rotational decoupling. In the following sections we will explain these observations in terms of the structure of confined water.

3.2. Structure of Confined Water. We study the structure of confined water by computing the density and number of HBs per water molecule as a function of distance from the confining surfaces for all surface polarities and the two wall separations.

3.2.1. Hydrogen Bonding. The number of $\mathrm{HBs}$ between water molecules and between water molecules and surface silanol groups (except for the case of non-hydroxylated silica, $k=0.0)$ is computed using a geometric definition based on that in ref 59, according to which a HB exists between two water molecules whenever the distance between oxygen atoms is less than $R_{\mathrm{OO}}=0.36 \mathrm{~nm}$ (the range of water's first coordination shell ${ }^{60}$ ) and the dimer angle (the angle formed by the vector $\vec{R}_{\mathrm{OO}}$ and the $\mathrm{O}-\mathrm{H}$ vector of the $\mathrm{H}$-donor), $\varphi$, is less than $30^{\circ}$. The dimer angle cutoff is obtained from the amplitude of the librations that break hydrogen bonds, which have been determined to be $\sim 30^{\circ}$. ${ }^{59}$ In the calculation of surface water $\mathrm{HBs}$, we consider the fact that surface $\mathrm{OH}$ groups only have one H-donor site and apply the same geometric criteria outlined above.

Figure 6 presents the total number of hydrogen bonds per water molecule, $\left\langle\mathrm{HB}_{\mathrm{Total}}(z)\right\rangle$, as a function of distance from the 


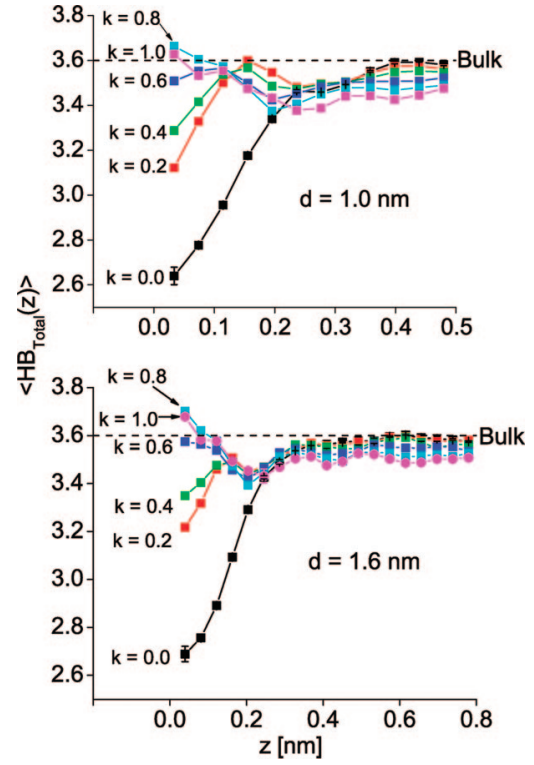

Figure 6. Total average number of hydrogen bonds (HB) per water molecule as a function of distance from the surfaces, $z . T=300 \mathrm{~K}, \rho$ $=1.0 \mathrm{~g} \mathrm{~cm}^{-3}$. Upper: $d=1.0 \mathrm{~nm}$, lower: $d=1.6 \mathrm{~nm}$.

surface for all surface polarities and wall separations. $\left\langle\mathrm{HB}_{\text {Total }}(z)\right\rangle$ was computed dividing the total number of hydrogen bonds by the number of water molecules present in $0.041 \mathrm{~nm}$ slabs parallel to the surfaces. The data presented are averages over $100 \mathrm{ps}$. Unless otherwise denoted in the figures, error bars in HB data are roughly the same size as the symbols in Figure 6. Both systems depicted in Figure 6 exhibit an average number of hydrogen bonds about 3.6 per water molecule near the center $(z=0.5$ and $0.8 \mathrm{~nm}$ for $d=1.0 \mathrm{~nm}$ and $d=1.6 \mathrm{~nm}$, respectively), in agreement with our bulk water simulations at $300 \mathrm{~K}$ and $\rho=1 \mathrm{~g} \mathrm{~cm}^{-3}$ and previous simulation studies. ${ }^{33}$ The limiting, bulk-like number of hydrogen bonds per water molecule shown in Figure 6 is consistent with the conventional picture of water as forming a transient tetrahedrally coordinated network, and at variance with the controversial interpretation of recent X-ray absorption and X-ray emission experiments, ${ }^{61,62}$ according to which most water molecules form structures in which they participate in only two hydrogen bonds.

In Figure 6, the number of hydrogen bonds per molecule near the silica surface decreases from $\sim 3.6$ at $k=1.0$ to 2.7 at $k=$ 0.0 , indicating that the HB network connectivity is compromised near the low polarity surfaces. We observe that for approximately $k \leq 0.4,\left\langle\mathrm{HB}_{\text {Total }}(z)\right\rangle \leq 3.6$ near the surfaces. In a previous study ${ }^{34}$ we showed that our surfaces are hydrophobic (i.e., they exhibit a contact angle $>90^{\circ}$ ) when $k \leq 0.4$. Therefore, the surface hydrophobicity, as measured by the contact angle, is directly related to the decrease in $\left\langle\mathrm{HB}_{\text {Total }}(z)\right\rangle$ near the surfaces.

In Figure 7 we present the average number of water-water (upper panel) and water-silanol hydrogen bonds (lower panel), which, added, result in the total number of hydrogen bonds given in Figure 6. We show only the results for $d=1.6 \mathrm{~nm}$ noting that those for $d=1.0 \mathrm{~nm}$ are qualitatively similar. The upper panel in Figure 7 shows that the number of water-water hydrogen bonds near the surface decreases monotonically with increasing $k$. The decrease in water-water hydrogen bonding is steeper for $k \geq 0.6$. Meanwhile, water-silanol hydrogen bonding (lower panel in Figure 7) increases with polarity for all $k$ values except for $k=0.0$, where hydrogen bonding with the surface is not possible. We observe that SPC/E water forms 2.7 hydrogen bonds per molecule near the apolar $(k=0.0)$ surface, in agreement with MD simulations of the water-vapor
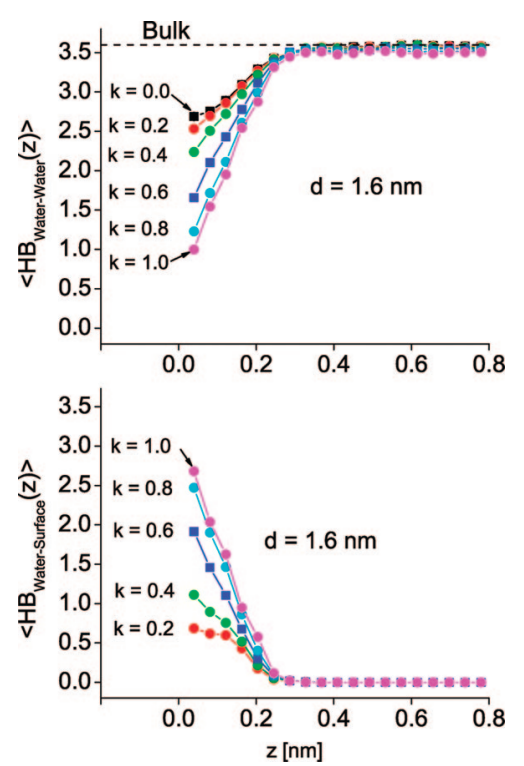

Figure 7. Average number of water-water (upper panel) and water-surface (lower panel) hydrogen bonds per water molecule as a function of distance from the surfaces, $z . T=300 \mathrm{~K}, \rho=1.0 \mathrm{~g} \mathrm{~cm}^{-3}$, $d=1.6 \mathrm{~nm}$.

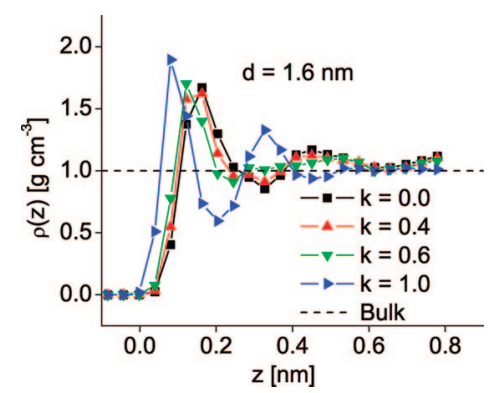

Figure 8. Density profile, $\rho(z)$ (local density in $0.041 \mathrm{~nm}$-thick slabs parallel to the surfaces) for different surface polarities. $T=300 \mathrm{~K}, \rho$ $=1.0 \mathrm{~g} \mathrm{~cm}^{-3}, d=1.6 \mathrm{~nm}$.

interface, ${ }^{33}$ a prototypical hydrophobic surface. ${ }^{63}$ Figure 7 (lower panel) also shows that surface-water hydrogen bonds only exist within $\sim 0.3 \mathrm{~nm}$ from the surface hydrogen.

The trends in Figure 7 therefore show that for $k \geq 0.2$, water-water HBs lost near the wall can be replaced by watersilanol HBs to an extent dictated by wall polarity. Water in contact with the hydrophobic apolar surface $(k=0.0)$ experiences a loss of $1 \mathrm{HB}$ near the surface, gaining none since the surface is not hydroxylated. Water molecules also show a net loss in HB connectivity near the $k=0.2$ and $k=0.4$ surfaces. Water molecules confined between surfaces with $k \geq 0.6$, on the other hand, are able to fully recover broken water-water hydrogen bonds given the strong electrostatic interactions that molecules establish with silanol groups.

3.2.2. Local Density. The local density as a function of distance from the surfaces, $\rho(z)$, is computed to study the extent of structural inhomogeneities introduced by confinement. The quantity $\rho(z)$ represents the local density in slabs of thickness $0.041 \mathrm{~nm}$ parallel to the confining surfaces. The density profile for $d=1.6 \mathrm{~nm}$ is presented in Figure 8 where, for the sake of clarity, we have omitted the curves corresponding to $k=0.2$ and $k=0.8$.

Figure 8 shows that the surfaces induce density oscillations that extend throughout the confined volume. Only the fluid confined by surfaces with $k=1.0$ appears to converge to a bulk density of $1.0 \mathrm{~g} \mathrm{~cm}^{-3}$. Density oscillations extending 

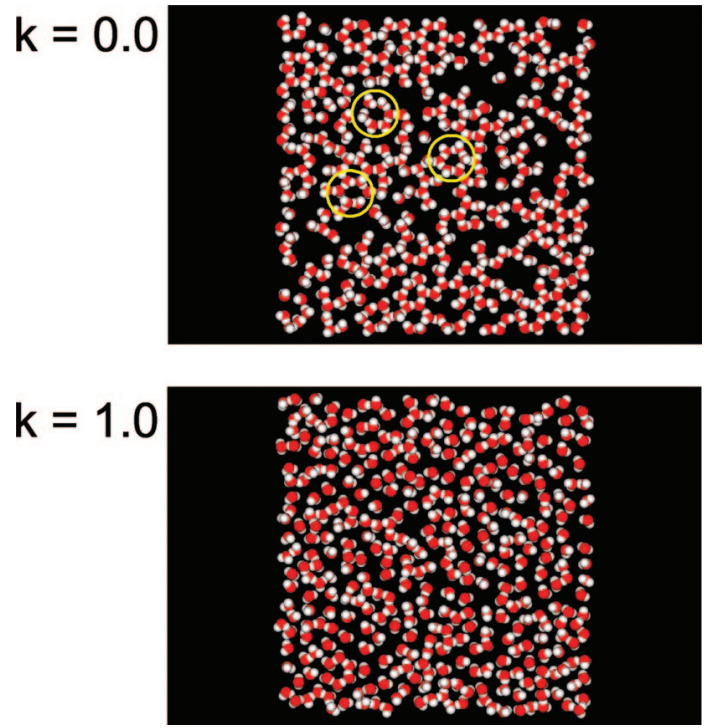

Figure 9. Front snapshot of the first layer of water molecules (layer thickness $=0.25 \mathrm{~nm})$ at the interface with a hydrophobic apolar $(k=$ 0.0 , upper panel) and a hydrophilic surface $(k=1.0$, lower panel), at $t=1.0 \mathrm{~ns}$, showing the presence of hexagonal structures (in yellow circles) on the apolar surface. $T=300 \mathrm{~K}, \rho=1.0 \mathrm{~g} \mathrm{~cm}^{-3}, d=1.6$ $\mathrm{nm}$.

throughout the confined volume are also observed at $d=1.0$ $\mathrm{nm}$ (data not shown). Figure 8 also shows that, as expected, an increase in surface polarity $k$ is accompanied by an increase in water density near the surfaces, manifested by the first peak in the density profile (that located at $z \leq 0.2 \mathrm{~nm}$ in Figure 8) moving closer to the interface.

3.3. Relation between Dynamics and Interfacial Water Structure. In this section we make the connection between confined water structure and local dynamics, based on the structural characterization presented above.

For water confined between surfaces with $k=1.0$, the slow translational and rotational dynamics are due to the high density of water near the surfaces (Figure 8) and the strong surface water HBs (Figure 7). The slow dynamics observed near the apolar surface $(k=0.0)$, on the other hand, are explained by the highly ordered water found at the interface. In Figure 9 we show snapshots of water molecules located within $0.25 \mathrm{~nm}$ from the surface located at $z>0$. The top panel shows that water at the apolar surfaces is ordered into hexagonal ice-like structures (some of which are shown in yellow circles in Figure 9). We observe the existence of such ice-like structures throughout the data collection. On the contrary, the lower panel in Figure 9, depicting water structure at the hydrophilic interface $(k=1.0)$, shows a dense but more disordered arrangement. The slow dynamics observed on our atomically detailed apolar surfaces are in contrast with the fast dynamics observed near smooth hydrophobic surfaces. ${ }^{29,47}$ This observation highlights the importance of template structure as a key factor, alongside surface polarity, confinement geometric length scale, temperature and density, in determining interfacial dynamics. For the case of $\beta$-cristobalite, different structural properties (number of siloxane bonds, surface defects) have been shown to significantly alter water adsorption, ${ }^{44}$ therefore suggesting that hydrophobic slowing down is an effect specific to the surface structure used in our simulations. It should be noted, however, that other hydrophobic surfaces may be able to induce crystallization of water under nanoscale confinement. For example, evidence of the room-temperature formation of monocrystalline water

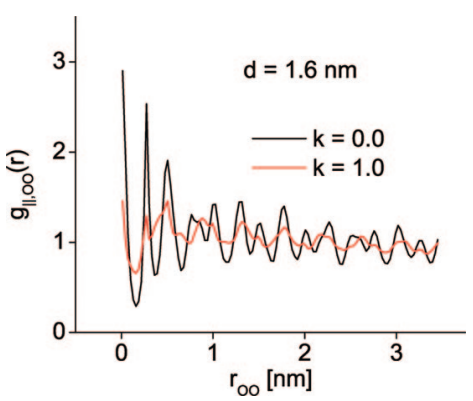

Figure 10. In-plane water oxygen-water oxygen radial distribution function, $g_{\|, \mathrm{OO}}(r)$, for the layer of water molecules within $0.3 \mathrm{~nm}$ from the interface, computed for $k=0.0$ and 1.0. $T=300 \mathrm{~K}, \rho=1.0 \mathrm{~g}$ $\mathrm{cm}^{-3}, d=1.6 \mathrm{~nm}$.

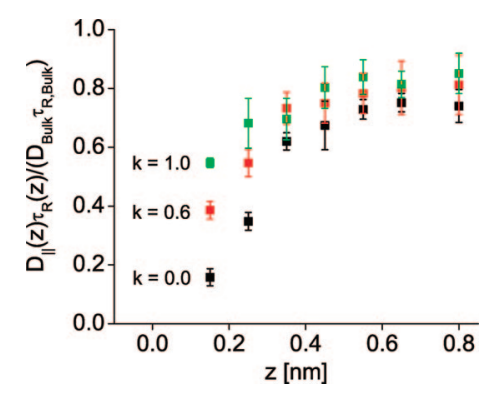

Figure 11. Product of the local in-plane diffusion coefficient and the rotational relaxation time, normalized by the bulk $D$ and $\tau_{\mathrm{R}}$, as a function of distance from the surfaces and various surface polarities. $T$ $=300 \mathrm{~K}, \rho=1.0 \mathrm{~g} \mathrm{~cm}^{-3}, d=1.6 \mathrm{~nm}$.

between a graphite surface and the tip of a friction force microscope was recently reported. ${ }^{20}$

The formation of the hexagonal structures is explained through a mechanism analogous to that of the templation of a bilayer ice confined between finite hydrophobic $\beta$-cristobalite surfaces at a wall-wall separation of $0.6 \mathrm{~nm} .{ }^{18}$ The hexagonal structures observed in Figure 9 (upper panel) are a consequence of Lennard-Jones interactions between water oxygen atoms and surface atoms ( $\mathrm{Si}$ and $\mathrm{O}$ ) on $\beta$-cristobalite, the morphology of which induces the formation of an ordered water layer. Through Lennard-Jones interactions water molecules arrange into hexagonal structures around surface oxygen atoms (cf. Figure $6 \mathrm{a}$ in ref 18). These structures exhibit slow dynamics because of the Lennard-Jones interactions with surface atoms and given that the presence of defects in the HB network (i.e., overcoordinated water molecules), which can "catalyze" molecular relaxation, ${ }^{24,64}$ is compromised at the apolar interface, where both local density and HB connectivity are low. A similar model of interfacial roughness was employed to explain slow HB dynamics near the (hydrophobic) water-dichloroethane liquidliquid interface. ${ }^{36}$ In this system, density fluctuations are responsible for creating "finger-like" water structures that protrude into the organic phase and result in water molecules with a lack of non-hydrogen-bonded water neighbors.

To provide a proof of the order imposed by the apolar surface on the first layer of water molecules, we compute the water oxygen-water oxygen in-plane radial distribution function (RDF) of the first layer of water molecules (Figure 10). Only the data for $d=1.6 \mathrm{~nm}$ are shown since identical conclusions are reached for $d=1.0 \mathrm{~nm}$. Figure 10 shows that the peaks corresponding to the nearest and next-nearest neighbors are located at $\sim 0.3 \mathrm{~nm}$ and $\sim 0.5 \mathrm{~nm}$, respectively, in agreement with the location of the nearest and next-nearest oxygen neighbors in a perfect hexagonal arrangement. ${ }^{18} \mathrm{We}$ also show the $\mathrm{RDF}$ for $k=1.0$, where the smaller peaks are indicative of 
much weaker position correlations, in agreement with the observations made about Figure 9 (lower panel). In fact, we note that the height and depth of the RDF peaks for the intermediate polarities (not shown) decrease monotonically with increasing $k$. This indicates that surface hydrogen atoms, present for $k \geq 0.2$, exert a structure-breaking effect.

The nonmonotonic evolution of $D_{\|}(z)$ and $\tau_{\mathrm{R}}(z)$ observed as $k$ is increased (Figures 3 and 5) is due to structural changes imposed by the presence of (charged) surface hydrogen atoms. When $k \geq 0.2$, surfaces are provided with $\mathrm{OH}$ groups that establish electrostatic interactions with water molecules. This in turn results in an increase in water density at the interface and the number of $\mathrm{HB}$ network defects that favor relaxation. Additionally, surface hydrogen atoms can reorient in a circle while interacting with nearby water molecules, thereby exerting a structure-breaking effect on interfacial water, as seen in Figure 10 , and in the absence of hexagonal structures at $k=1.0$ (Figure 9 , lower panel). The disruption in water ordering by surface $\mathrm{OH}$ groups explains the increase in translational mobility for $k$ $\in[0.2,0.8]$. Upon further increasing surface polarity to $k=1.0$, we observe a sharp increase in water density near the surfaces (Figure 8), caused by strong electrostatic attraction between the surfaces and water. The high water density and strong surface-water HBs at the fully hydrophilic surface result in hindered molecular motion.

3.4. Translational-Rotational Decoupling. In a bulk fluid at temperatures well above the glass transition, translational and rotational dynamics are well described by the Stokes-Einstein (SE) and Stokes-Einstein-Debye (SED) relations, ${ }^{65,66}$ presented in eqs 8 and 9 , respectively,

$$
\begin{gathered}
D=\frac{k_{\mathrm{B}} T}{6 \pi \eta r} \\
\tau_{\mathrm{R}}=\frac{4 \pi \eta r^{3}}{3 k_{\mathrm{B}} T}
\end{gathered}
$$

where $k_{\mathrm{B}}$ is Boltzmann's constant, $T$ the temperature, $\eta$ the viscosity, and $r$ the molecular "radius". The temperature and viscosity dependencies of the SE and SED relations are such that their product, $D \tau_{\mathrm{R}}$, a quantity analogous to the ratio of $D$ to the rotational diffusion coefficient, is a constant dependent only on $r^{2}$. Whenever this ratio deviates from constant behavior, the system is said to exhibit translational-rotational decoupling. 56

As pointed out in section 3.1.2, rotational and translational dynamics of our confined water systems appear to be decoupled. In Figure 11, we investigate this phenomenon locally, plotting the product $D_{\|}(z) \tau_{\mathrm{R}}(z)$, normalized by $D_{\mathrm{Bulk}} \tau_{\mathrm{R}, \mathrm{Bulk}}$, as a function of $z$. For clarity, we only show the data for $k=0.0,0.6$, and 1.0 , and $d=1.6 \mathrm{~nm}$. For all $k$ and $z$, the product $D_{\|}(z) \tau_{\mathrm{R}}(z)$ is smaller than $D_{\text {Bulk }} \tau_{\mathrm{R}, \text { Bulk }}$, indicating that confinement induces a more pronounced slowing down of translational dynamics. The dynamic decoupling is accentuated near the surfaces, where $D_{\|}(z) \tau_{\mathrm{R}}(z)$ decreases further. Experiments and simulations by Ladanyi and collaborators ${ }^{57}$ found a more pronounced slowing down of translational dynamics relative to rotational in water confined inside reverse micelles. Figure 11 also shows that the extent of decoupling is dependent on surface polarity: at constant $z$, the value of $D_{\|}(z) \tau_{\mathrm{R}}(z)$ increases monotonically with $k$ within $\sim 0.3 \mathrm{~nm}$ from the surfaces. This is caused by a lower diffusion coefficient near the apolar and low polarity surfaces, as can be observed in Figure 3, which shows that the smallest values of $D_{\|}(z)$ occur at $k \leq 0.4$.

In order to explain the decoupling we refer to the $\mathrm{HB}$ and density profiles in Figures 6 and 8. Figure 6 shows that HB connectivity per molecule confined by the hydrophilic surfaces $(k \geq 0.6)$ remains fairly constant around the bulk value, approaching $\sim 3.6$ hydrogen bonds per molecule at the center. $\left\langle\operatorname{HB}_{\text {Total }}(z)\right\rangle$ for the apolar and weakly polar surfaces reaches the bulk value at a distance of $\sim 0.3 \mathrm{~nm}$ from the surfaces. In regard to the local density, Figure 8 shows that the oscillations in $\rho(z)$ decay in amplitude with distance from the surfaces but remain visible for all values of $z$. Figures 6 and 8 therefore suggest that the perturbations due to confinement affect the local density more significantly and allow us to explain the decoupling as follows. It is known that the rotational relaxation of water is related to the breaking and reforming of hydrogen bonds. ${ }^{64,67}$ Our data show that the number of hydrogen bonds per molecule is only moderately perturbed by confinement, attaining bulklike connectivity for $z \geq 0.3 \mathrm{~nm}$. This bulk-like hydrogenbonding environment results in bulk-like rotational relaxation times for molecules located far from the surfaces. Conversely, Figure 8 shows that the confining surfaces significantly perturb the liquid density, with the system with $k=1.0$ and $d=1.6$ $\mathrm{nm}$ being the only one showing convergence toward a bulk density of $1.0 \mathrm{~g} \mathrm{~cm}^{-3}$. The regions of high density shown in Figure 8 explain why $D_{\|}(z)$ is lower than the bulk diffusion coefficient, given that the diffusion coefficient of water decreases with increasing density at ambient temperature. ${ }^{68}$

\section{Conclusions}

We have investigated the effect of surface polarity, $k$, of $\beta$-cristobalite $\{111\}$ planes on the structure and dynamics of water in confinement. We have characterized the structure of water in our model through the average number of hydrogen bonds per molecule and local density profile. The computed density profiles (Figure 8) confirm that confined water is an inhomogeneous fluid for all surface polarities and wall separations investigated. Our results for the number of hydrogen bonds per molecule (Figure 6) show that HB network connectivity is only compromised near the apolar and slightly polar surfaces ( $k \leq 0.4$ ), reaching the bulk-like value of $\sim 3.6$ hydrogen bonds per molecule at $z \geq 0.3 \mathrm{~nm}$ from the surfaces.

We find that rotational and translational dynamics (Figures 3 and 5) evolve nonmonotonically with polarity. For molecules close to the surfaces, rotational and translational mobilities are maximized at $k=0.6$ and $k=0.8$, respectively. These phenomena are explained by the different structural features of water near hydrophobic and hydrophilic surfaces. In the case of hydrophobic apolar confinement $(k=0.0), \beta$-cristobalite induces the formation of short-lived ice-like structures (Figure 9) exhibiting hindered dynamics. Surface hydrogens, occurring when $k \geq 0.2$, disrupt water surface ordering (Figure 10) and enhance translational and rotational dynamics. Increasing the polarity to $k=1.0$ results in a region of high water density adjacent to the surfaces (Figure 8). The increased number of molecules at the fully hydrophilic surface, accompanied by the formation of stronger hydrogen bonds between water and surface $\mathrm{OH}$ groups, hinders molecular motion.

In addition, we find evidence for translational-rotational decoupling, characterized by a more drastic decrease of translational dynamics near the confining surfaces relative to the corresponding rotational motion. We observe that the diffusion coefficient at the center is lower than its bulk counterpart, while the rotational relaxation time is bulk-like at 
the center of the system with $d=1.6 \mathrm{~nm}$ and also for the systems with polarities $k=0.4$ and 0.6 at $d=1.0 \mathrm{~nm}$. We explain this result by the dependence of $D_{\|}(z)$ on local density, which experiences a more extensive perturbation due to the confining surfaces compared with the HB network connectivity, which determines $\tau_{\mathrm{R}}(z)$.

Our results emphasize the importance of template structure as a key factor in the study of water dynamics in nanoscopic confinement, pointing out some possible directions for future inquiry. Within the specific context of silica-confined water films, the dynamics of water confined by amorphous surfaces, as well the inclusion of surface defects in crystalline silica ( $\alpha$ and $\beta$-cristobalite) surfaces, pose interesting questions that warrant future studies.

Acknowledgment. Financial support for this work was provided by MRSEC NSF (DMR-0213706) and ARO-MURI (W911NF-04-1-0170).

\section{References and Notes}

(1) Ball, P. Chem. Rev. 2008, 108, 74-108.

(2) Cheng, Y. K.; Rossky, P. J. Nature 1998, 392 (6677), 696-699.

(3) Hua, L.; Huang, X. H.; Liu, P.; Zhou, R. H.; Berne, B. J. J. Phys. Chem. B 2007, 111, 9069-9077.

(4) Zhou, R. H.; Huang, X. H.; Margulis, C. J.; Berne, B. J. Science 2004, 305 (5690), 1605-1609.

(5) van Hijkoop, V. J.; Dammers, A. J.; Malek, K.; Coppens, M. O. J. Chem. Phys. 2007, 127 (8), 085101.

(6) Zhu, F. Q.; Schulten, K. Biophys. J. 2003, 85 (1), 236-244.

(7) Kalra, A.; Garde, S.; Hummer, G. Proc. Natl. Acad. Sci. U.S.A 2003, 100 (18), 10175-10180

(8) Hummer, G.; Rasaiah, J. C.; Noworyta, J. P. Nature 2001, 414 (6860), 188-190.

(9) Austin, R. Nature Nanotechnol. 2007, 2 (2), 79-80

(10) Bagchi, B. Chem. Rev. 2005, 105, 3197-3219.

(11) McGrath, K. M.; Dabbs, D. M.; Yao, N.; Aksay, I. A.; Gruner, S. M. Science 1997, 277 (5325), 552-556.

(12) Klapp, S., Schoen, M., Eds.; Reviews in Computational Chemistry; John Wiley \& Sons: Hoboken, NJ, 2007; Vol. 24.

(13) Christenson, H. K.; Claesson, P. M. Science 1988, 239 (4838), 390392

(14) Jensen, T. R.; Jensen, M. O.; Reitzel, N.; Balashev, K.; Peters, G. H.; Kjaer, K.; Bjornholm, T. Phys. Rev. Lett. 2003, 90 (8), 086101.

(15) Bratko, D.; Curtis, R. A.; Blanch, H. W.; Prausnitz, J. M. J. Chem. Phys. 2001, 115 (8), 3873-3877.

(16) Ruan, C. Y.; Lobastov, V. A.; Vigliotti, F.; Chen, S. Y.; Zewail, A. H. Science 2004, 304 (5667), 80-84.

(17) Asay, D. B.; Kim, S. H. J. Phys. Chem. B 2005, 109, 16760-16763.

(18) Giovambattista, N.; Rossky, P. J.; Debenedetti, P. G. Phys. Rev. E 2006, 73 (4), 041604.

(19) Zangi, R. J. Phys.-Condens. Matter 2004, 16 (45), S5371-S5388. (20) Jinesh, K. B.; Frenken, J. W. M. Phys. Rev. Lett. 2008, 101 (3), 036101

(21) Goel, G.; Krekelberg, W. P.; Errington, J. R.; Truskett, T. M. Phys. Rev. Lett. 2008, 100 (10), 106001.

(22) Verdaguer, A.; Sacha, G. M.; Bluhm, H.; Salmeron, M. Chem. Rev. 2006, 106, 1478-1510.

(23) Shen, Y. R.; Ostroverkhov, V. Chem. Rev. 2006, 106, 1140-1154. (24) Rezus, Y. L. A.; Bakker, H. J. Phys. Rev. Lett. 2007, 99 (14), 148301 .

(25) Persson, E.; Halle, B. Proc. Natl. Acad. Sci. U.S.A. 2008, 105 (17), 6266-6271.

(26) Tehei, M.; Franzetti, B.; Wood, K.; Gabel, F.; Fabiani, E.; Jasnin, M.; Zamponi, M.; Oesterhelt, D.; Zaccai, G.; Ginzburg, M.; Ginzburg, B. Z. Proc. Natl. Acad. Sci. U.S.A. 2007, 104 (3), 766-771.

(27) Ostroverkhov, V.; Waychunas, G. A.; Shen, Y. R. Phys. Rev. Lett. 2005, 94 (4), 046102. 532.

(28) Frank, H. S.; Evans, M. W. J. Chem. Phys. 1945, 13 (11), 507-

(29) Lee, S. H.; Rossky, P. J. J. Chem. Phys. 1994, 100 (4), 33343345
(30) Marti, J.; Nagy, G.; Gordillo, M. C.; Guardia, E. J. Chem. Phys. 2006, 124 (9), 094703.

(31) Gallo, P.; Rovere, M. J.Phys.-Condens. Matter 2003, 15 (45), $7625-7633$.

(32) Giovambattista, N.; Debenedetti, P. G.; Rossky, P. J. J. Phys. Chem. C 2007, 111, 1323-1332.

(33) Liu, P.; Harder, E.; Berne, B. J. J. Phys. Chem. B 2005, 109, 29492955.

(34) Giovambattista, N.; Debenedetti, P. G.; Rossky, P. J. J. Phys. Chem. B 2007, 111, 9581-9587.

(35) Balasubramanian, S.; Pal, S.; Bagchi, B. Phys. Rev. Lett. 2002, 89 (11), 115505.

(36) Benjamin, I. J. Phys. Chem. B 2005, 109, 13711-13715.

(37) Pal, S.; Bagchi, B.; Balasubramanian, S. J. Phys. Chem. B 2005, 109, 12879-12890.

(38) Berendsen, H. J. C.; Postma, J. P. M.; Vangunsteren, W. F.; Dinola, A.; Haak, J. R. J. Chem. Phys. 1984, 81 (8), 3684-3690.

(39) Berendsen, H. J. C.; Grigera, J. R.; Straatsma, T. P. J. Phys. Chem. 1987, 91, 6269-6271.

(40) van der Spoel, D.; van Maaren, P. J.; Berendsen, H. J. C. J. Chem. Phys. 1998, 108 (24), 10220-10230.

(41) Berendsen, H. J. C.; Postma, J. P. M.; van Gunsteren, W. F.; Hermans, J. In Intermolecular Forces; Pullman, B., Ed.; Reidel: Dordrecht, The Netherlands, 1981; pp 331-342.

(42) Jorgensen, W. L.; Chandrasekhar, J.; Madura, J. D.; Impey, R. W.;

Klein, M. L. J. Chem. Phys. 1983, 79 (2), 926-935.

(43) Sosman, R. B. The Phases of Silica; Rutgers University Press: New Brunswick, NJ, 1965.

(44) Nangia, S.; Washton, N. M.; Mueller, K. T.; Kubicki, J. D.; Garrison, B. J. J. Phys. Chem. C 2007, 111, 5169-5177.

(45) McQuarrie, D. A. Statistical Mechanics; Harper \& Row: New York, 1976.

(46) Liu, P.; Harder, E.; Berne, B. J. J. Phys. Chem. B 2004, 108, 65956602

(47) Kumar, P.; Buldyrev, S. V.; Starr, F. W.; Giovambattista, N.; Stanley, H. E. Phys. Rev. E 2005, 72 (5), 051503.

(48) Scheidler, P.; Kob, W.; Binder, K. Europhys. Lett. 2002, 59 (5), 701-707.

(49) Mark, P.; Nilsson, L. J. Comput. Chem. 2002, 23 (13), 1211-1219. (50) Heyes, D. M. J. Chem. Soc., Faraday Trans. 1994, 90 (20), 30393049

(51) Temperley, H. N. V., Rowlinson, J. S., Rushbrooke, G. S., Eds. Physics of Simple Liquids; North-Holland Publishing Co.: Amsterdam, 1968.

(52) Sega, M.; Vallauri, R.; Melchionna, S. Phys. Rev. E 2005, 72 (4), 041201.

(53) Sciortino, F.; Gallo, P.; Tartaglia, P.; Chen, S. H. Phys. Rev. E 1996, 54 (6), 6331-6343. 267.

(54) Debenedetti, P. G.; Stillinger, F. H. Nature 2001, 410 (6825), 259-

(55) Mazza, M. G.; Giovambattista, N.; Stanley, H. E.; Starr, F. W. Phys. Rev. E 2007, 76 (3), 031203.

(56) Lombardo, T. G.; Debenedetti, P. G.; Stillinger, F. H. J. Chem. Phys. 2006, 125 (17), 174507.

(57) Harpham, M. R.; Ladanyi, B. M.; Levinger, N. E.; Herwig, K. W. J. Chem. Phys. 2004, 121 (16), 7855-7868.

(58) Cicerone, M. T.; Ediger, M. D. J. Chem. Phys. 1996, 104 (18), $7210-7218$.

(59) Luzar, A.; Chandler, D. Phys. Rev. Lett. 1996, 76 (6), 928-931. (60) Marti, J.; Padro, J. A.; Guardia, E. J. Chem. Phys. 1996, 105 (2), 639-649.

(61) Wernet, P.; Nordlund, D.; Bergmann, U.; Cavalleri, M.; Odelius, M.; Ogasawara, H.; Naslund, L. A.; Hirsch, T. K.; Ojamae, L.; Glatzel, P.; Pettersson, L. G. M.; Nilsson, A. Science 2004, 304 (5673), 995-999.

(62) Tokushima, T.; Harada, Y.; Takahashi, O.; Senba, Y.; Ohashi, H.;

Pettersson, L. G. M.; Nilsson, A.; Shin, S. Chem. Phys. Lett. 2008, 460 (4-6), 387-400.

(63) Stillinger, F. H. J. Solution Chem. 1973, 2 (2/3), 141-158.

(64) Sciortino, F.; Geiger, A.; Stanley, H. E. Nature 1991, 354 (6350), 218-221.

(65) Einstein, A. Investigations on the Theory of Brownian Motion; Dover Publications: New York, 1956.

(66) Debye, P. Polar Molecules; Dover Publications: New York, 1945.

(67) Laage, D.; Hynes, J. T. Science 2006, 311 (5762), 832-835.

(68) Starr, F. W.; Sciortino, F.; Stanley, H. E. Phys. Rev. E 1999, 60 (6), 6757-6768.

JP809032N 